\title{
Management and Outcome of Periprocedural Cardiac Perforation and Tamponade with Radiofrequency Catheter Ablation of Cardiac Arrhythmias: A Single Medium-Volume Center Experience
}

\author{
Nebojša Mujović · Milan Marinković · Nebojša Marković · Aleksandar Kocijančić • \\ Vladan Kovačević · Dragan Simić · Arsen Ristić · Goran Stanković · Biljana Miličić · \\ Svetozar Putnik · Bosiljka Vujisić-Tešić · Tatjana S. Potpara
}

Received: July 6, 2016 / Published online: August 23, 2016

(c) The Author(s) 2016. This article is published with open access at Springerlink.com

\begin{abstract}
Introduction: Cardiac tamponade (CT) is a life-threatening complication of radiofrequency ablation (RFA). The course and outcome of CT in low-to-medium volume electrophysiology centers are underreported.
\end{abstract}

Enhanced content To view enhanced content for this article go to http://www.medengine.com/Redeem/3295 F0604A1B548E.

N. Mujović · M. Marinković · N. Marković .

A. Kocijančić · V. Kovačević · D. Simić · A. Ristić .

G. Stanković · B. Vujisić-Tešić · T. S. Potpara

Cardiology Clinic, Clinical Center of Serbia,

Belgrade, Serbia

N. Mujović $(\varangle) \cdot$ D. Simić · A. Ristić · G. Stanković ·

S. Putnik · B. Vujisić-Tešić · T. S. Potpara ( $\square)$

School of Medicine, University of Belgrade,

Belgrade, Serbia

e-mail: nmujovic@gmail.com

T. S. Potpara

e-mail: tanjapotpara@gmail.com

B. Miličić

Center for Anesthesia, Clinical Center of Serbia,

Belgrade, Serbia

B. Miličić

Faculty of Stomatology, University of Belgrade,

Belgrade, Serbia

S. Putnik

Clinic for Cardiac Surgery, Clinical Center of Serbia, Belgrade, Serbia
Methods: We analyzed the incidence, management and outcomes of CT in 1500 consecutive RFAs performed in our center during 2011-2016.

Results: Of 1500 RFAs performed in 1352 patients (age 55 years, interquartile range: 41-63), 569 were left-sided procedures $(n=406$ with transseptal access). Conventional RFA or irrigated RFA was performed in $40.9 \%$ and $59.1 \%$ of procedures, respectively. Ablation was performed mostly for atrioventricular nodal reentrant tachycardia $(25.4 \%)$, atrial fibrillation (AF; 18.5\%), atrial flutter (18.4\%), accessory pathway (16.5\%) or idiopathic ventricular arrhythmia (VA; $12.3 \%)$, and rarely for structural VA (2.1\%). CT occurred in 12 procedures (0.8\%): $10 \mathrm{AF}$ ablations, 1 idiopathic VA and 1 typical atrial flutter ablation. Factors significantly associated with CT were older age, pre-procedural oral anticoagulation, left-sided procedures, transseptal access, AF ablation, irrigated RFA and longer fluoroscopy time (on univariate analysis), and AF ablation (on multivariable analysis). The perforation site was located in the left atrium $(n=7)$, right atrium $(n=3)$, or in the left ventricle or coronary sinus $(n=1$ each $)$ Upon 
pericardiocentesis, two patients underwent urgent cardiac surgery because of continued bleeding. There was no fatal outcome. During the follow-up of $19 \pm 14$ months, eight patients were arrhythmia free.

Conclusion: Incidence of RFA-related CT in our medium-volume center was low and significantly associated with AF ablation. The outcome of CT was mostly favorable after pericardiocentesis, but readily accessible cardiothoracic surgery back-up should be mandatory in RFA centers.

Keywords: Atrial fibrillation; Cardiac perforation; Cardiac tamponade; Cardiology; Catheter ablation; Outcomes; Procedure-related complications

\section{INTRODUCTION}

Electrophysiology (EP) development has been accompanied by an exponential increase in the number of catheter ablation (CA) procedures [1]. Although the CA success rates have improved, the rate of complications has remained unchanged, probably because of an increase in the number of complex procedures $[1,2]$.

Cardiac perforation with tamponade is the most frequent potentially fatal complication of CA, with the incidence of $0.2-6 \%[3,4]$. Death and pericardiocentesis-related complications are twice higher with tamponades occurring during cardiac interventional procedures than with tamponade caused by other factors [5]. Additionally, the CA procedure discontinuation due to tamponade is often clinically frustrating, as it usually implies the need to repeat the procedure, which carries an additional psychological burden for both the patient and operator. The prevention of CA-related tamponade, its early detection and prompt management are of critical importance for the patient's outcome [5-8].

The number of reports on the management and outcomes of tamponade resulting from EP procedures is limited, and published data are mostly pertinent to high-volume academic centers, while reports from medium and small centers are scarce $[1,2,6-8]$. Given that low-to-medium-volume centers are the only available for CA of cardiac arrhythmias for many cardiology practices in all parts of the world, the data from a medium-volume center would be of interest for many general cardiologists when discussing CA with their patients. In this article, we describe the incidence, management and outcomes of CA-related cardiac perforation in a set of 1500 consecutive CA procedures performed in a single, medium-volume center over a 5-year period.

\section{METHODS}

We analyzed consecutive radiofrequency (RF) CA procedures performed between June 2011 and January 2016 in the Clinical Centre of Serbia. We retrospectively analyzed the data prospectively collected within the hospital EP laboratory electronic database and patient medical records. Before the ablation procedure, written informed consent was obtained in all patients. The study was conducted in accordance with the Declaration of Helsinki of 1964 as revised in 2013. The study protocol was approved by the Institutional Review Board of our hospital.

\section{Ablation Procedure}

Via the right femoral vein, three multipolar catheters were inserted into the distal coronary sinus (CS), right atrium (RA) and right ventricle 
(RV). During all of the procedures noninvasive measurement of arterial blood pressure was performed every $5 \mathrm{~min}$ along with continuous noninvasive pulse oximetry.

\section{Left-Sided Procedures}

Transseptal puncture (TSP) was carried out with a long needle and sheath (BRK1/BRK1-XS, Swartz SL0/SL1, Daig Corp., Minnetonka, MN, USA) under fluoroscopy in two orthogonal (the right and left anterior oblique $45^{\circ}$ ) projections and using only a CS catheter as an anatomical landmark, as reported previously [9]. Transesophageal (TEE) or intracardiac echocardiograms (ICE) were performed if needed, at the discretion of the operator. The right femoral artery was used for the transaortic approach. Ablation of left-sided accessory pathways (APs) and left-sided ventricular arrhythmias (VAs) was performed with the transseptal and/or transaortic approach, depending on the operator's preference.

\section{RF Ablation}

Ablation of supraventricular tachycardia and idiopathic VAs was performed using solid 4-mm conventional-tip catheters (RF Marinr, Medtronic, USA) or 3.5 to 4-mm externally irrigated-tip catheters (CoolPath or CoolFlex, St Jude, USA, or ThermoCool Biosense-Webster, USA), depending the operator's preference, while ablation of atrial flutter (AFL), atrial tachycardia (AT), atrial fibrillation (AF), and structural ventricular tachycardia (VT) was exclusively performed with irrigated $\mathrm{RF}$ technology. Ablation was performed in the temperature mode. For conventional ablations the setup was $55-65^{\circ} \mathrm{C}$ and $30-50 \mathrm{~W}$, while in irrigated $\mathrm{RF}$ ablations, it was $43^{\circ} \mathrm{C}$ and $30-40 \mathrm{~W}$ with a flow rate of $17-30 \mathrm{ml} / \mathrm{min}$. RF application was promptly terminated in the case of any increase in impedance or its decrease by $>10 \Omega$. The navigation mapping system (Velocity, St Jude or CARTO3 Biosense-Webster, USA) was used in all AF or structural VT ablations and in some AT and idiopathic VA ablations.

\section{AF Ablation}

The venous approach with three catheters was applied, one of which was inserted into the distal CS, for stimulation and electroanatomical reference. A single TSP was used, inserting two long sheaths through the same puncture site into the left atrium (LA). Navigation of the ablation catheter was performed with a long steerable sheath (8.5-Fr Agilis NXT, St Jude Medical, MN, USA), and pulmonary vein (PV) activity was evaluated with a circular 10- to 20-polar catheter navigated by a long non-steerable sheath (Swartz SL0 or SL1). After selective PV angiography, a 3D LA map was created, and fusion with the computed tomographic image was performed. In all patients with AF paroxysms lasting $<48 \mathrm{~h}$, only circumferential antral PV isolation was performed. The setup was $43^{\circ} \mathrm{C}$ and $30 \mathrm{~W}$. RF applications on the anterior and posterior wall lasted 40-60 s and 20-40 s, respectively. In the sites with PV activation sequence change, delay or isolation, RF application was prolonged to 90-120 s. In patients with prolonged AF episodes $(>48 \mathrm{~h})$, additional substrate ablation was performed. The complex atrial electrograms were visually identified, and their ablation was performed until local potential elimination was achieved. Endocardial LA roof and mitral isthmus ablation was performed using $30 \mathrm{~W}$, with the applications lasting 60-120 s in the same location, up to the linear block or maximum $30 \mathrm{~min}$ of RF per line. Epicardial mitral isthmus ablation in the distal CS was performed with RF pulses of 18-25 W lasting 40-120 s cumulatively up to $8 \mathrm{~min}$. 


\section{Anticoagulation}

Unfractionated heparin was administered intravenously during persistent AFL/AT ablation and in all left-sided procedures (bolus of 50-100 i.u./kg). In all patients receiving oral anticoagulant therapy, the strategy of interrupted warfarin or a non-vitamin $\mathrm{K}$ antagonist oral anticoagulant (NOAC) and bridging with subcutaneous enoxaparin (0.5 mg/kg, twice a day) was applied. The procedure was performed when the international normalized ratio (INR) was below 1.5. In patients taking antiplatelet therapy, ablation was performed without interrupting aspirin therapy, but not in case of dual antiplatelet therapy. During AF ablation, unfractionated heparin was administered immediately after TSP, with the maintenance of activated clotting time (ACT) in the 280-350 s range. Four hours after hemostasis, enoxaparin was reintroduced, which was also done the following day with the oral anticoagulant, with bridging until INR reached a value $\geq 2$.

\section{Pericardiocentesis}

In case of systolic arterial pressure dropping below $90 \mathrm{mmHg}$ and/or movement loss in the left margin of the cardiac silhouette during fluoroscopy, an echocardiogram was performed immediately (Fig. 1a, b). After the tamponade confirmation, intravenous fluid infusion and inotropic support were started. If the patient had received heparin, $50 \mathrm{mg}$ of protamine was administered intravenously.

The fluoroscopically guided subxiphoid approach was applied for pericardiocentesis in the left lateral projection [11]. The skin was punctured $2 \mathrm{~cm}$ below the xiphoid process with a needle positioned on a contrast-filled syringe and directed toward the left shoulder.
Intermittent injection of the contrast dye was used to confirm the progress of the needle through the tissue. Upon entering the pericardial space, $30-50 \mathrm{ml}$ of blood was drawn out, and $20 \mathrm{ml}$ of contrast dye was injected (Fig. 1c, d). Next, a long J-shaped guidewire was introduced through the needle into the pericardial cavity, a 6- or 8-Fr pigtail drainage catheter was positioned over the wire into the pericardial sac, and blood removal was continued. In case of continuous bleeding, direct auto-transfusion of the drained blood via a femoral venous sheath and/or allogeneic transfusion was performed, and the cardiothoracic surgical team was alerted. If definite hemodynamic stability was achieved using pericardiocentesis, $120 \mathrm{mg}$ of gentamicin and $100 \mathrm{mg}$ of methylprednisolone were injected via the drainage catheter into the pericardial cavity, and the patient was transferred to the Intensive Care Unit. Enoxaparin was restarted when the volume of the drained fluid fell below $20 \mathrm{ml}$ in $4 \mathrm{~h}$, while the oral anticoagulant was restarted after drainage catheter removal, usually $12-48 \mathrm{~h}$ upon pericardiocentesis.

\section{Study Outcomes}

The principal study outcome was cardiac perforation during CA. The perforation site was determined either directly during the surgical procedure or in other patients was assumed on the basis of the following: (1) catheter movement outside the cardiac silhouette during fluoroscopy; (2) loss of pace capture from the catheter tip; (3) audible "steam-pops" and/or sudden impedance increase during RF; (4) sudden hypotension after RF application; (5) contrast dye retention in the pericardium after a TSP attempt [8]. 

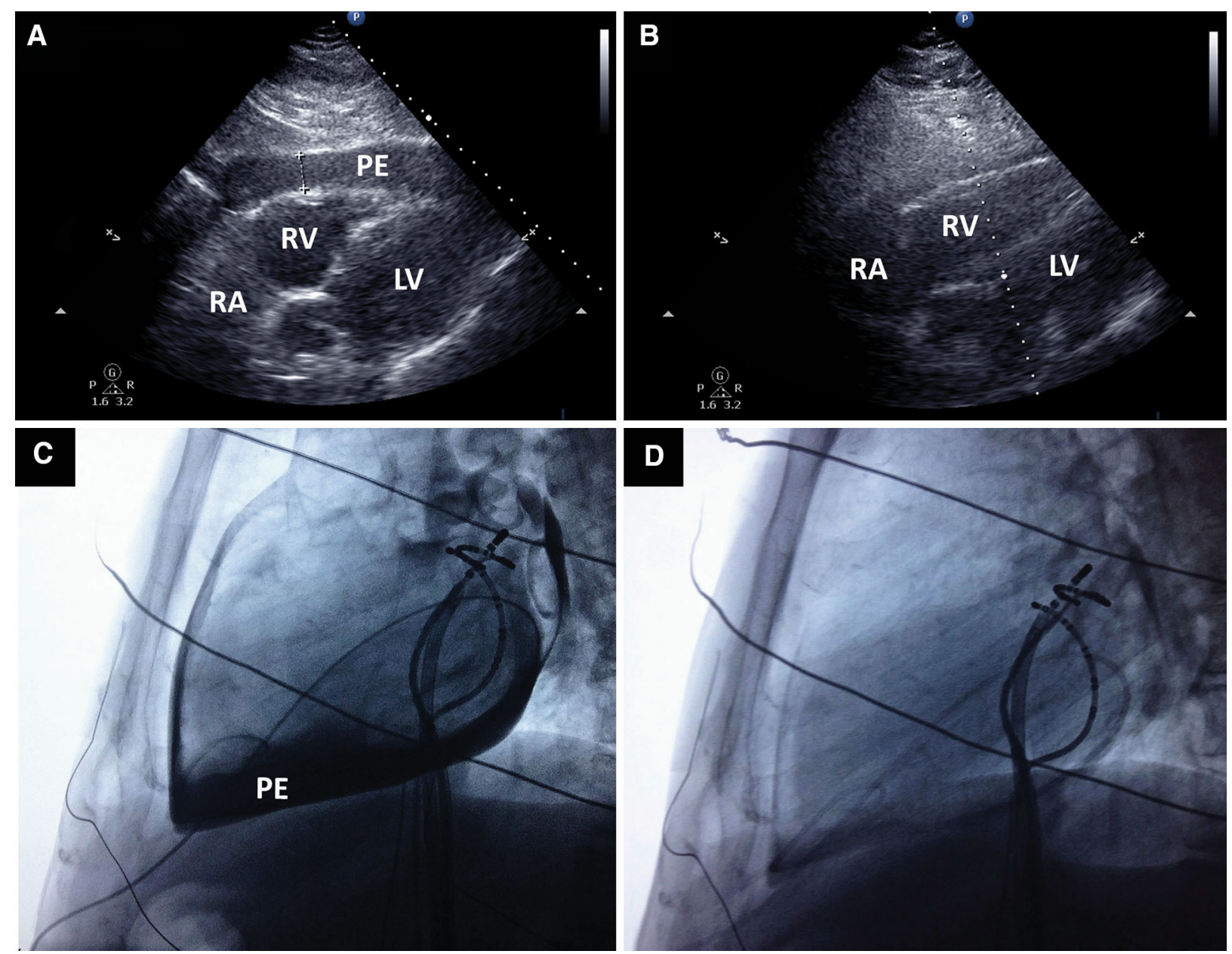

Fig. 1 Echocardiographic subxiphoid images (a, b) and left lateral fluoroscopic images $(\mathbf{c}, \mathbf{d})$ of pericardial effusion leading to cardiac tamponade, before and after

pericardiocentesis, respectively. $L V$ left ventricle, $P E$ pericardial effusion, $R A$ right atrium, $R V$ right ventricle

\section{Statistical Analysis}

Continuous variables are presented as mean $( \pm 1$ standard deviation) or median (with the interquartile range (IQR)], while categorical variables are summarized as percentages. The association of clinical parameters and procedure-related variables presented in Table 2 with the occurrence and outcome of tamponade was analyzed using univariate and multivariable binary logistic regression methods. A two-sided $P$ value of $<0.05$ was considered statistically significant. Analyses

were performed using the SPSS software, version 18.0 (IBM Corp., Armonk, NY, USA).

\section{RESULTS}

During the study period, 1500 consecutive RF-CA procedures were performed in 1352 patients (224 were redo procedures). The median patient age was 55 years (IQR: $41-63$ years), $58.1 \%$ were male, and $2.3 \%$ of all procedures were pediatric (i.e., performed in patients $<18$ years old). The distribution of CA procedure types is presented in Table 1 . A total 
Table 1 Baseline demographic characteristics

\begin{tabular}{|c|c|}
\hline Characteristics & $\begin{array}{l}\text { Total number } \\
\text { of procedures } \\
(n=1500)\end{array}$ \\
\hline Number of patients, $n$ & 1352 \\
\hline Age (years) & $55.0(41.0-63.0)$ \\
\hline Age $<18$ years & $34(2.3)$ \\
\hline Males & $872(58.1)$ \\
\hline $\operatorname{BMI}\left(\mathrm{kg} / \mathrm{m}^{2}\right)$ & $26.6(24.6-29.8)$ \\
\hline Heart failure & $238(15.9)$ \\
\hline Left ventricular EF (\%) & $61.0(56.0-66.0)$ \\
\hline LA diameter $(\mathrm{mm})$ & $38.0(34.0-43.0)$ \\
\hline Coronary artery disease & $174(11.6)$ \\
\hline Valvular heart disease & $37(2.5)$ \\
\hline Hypertension & $509(33.9)$ \\
\hline Diabetes mellitus & $111(7.4)$ \\
\hline Oral anticoagulation before procedure & $458(30.5)$ \\
\hline Number of antiarrhythmic drugs & $2.0(1.0-2.0)$ \\
\hline Redo procedure & $244(16.3)$ \\
\hline SVT (non-AF) & $1007(67.1)$ \\
\hline AVNRT & $381(25.4)$ \\
\hline Typical atrial flutter & $276(18.4)$ \\
\hline Accessory pathway & $248(16.5)$ \\
\hline Atrial tachycardia & $56(3.7)$ \\
\hline $\mathrm{AV}$ junction & $46(3.1)$ \\
\hline $\mathrm{AF}$ & $277(18.5)$ \\
\hline Paroxysmal AF & $208(75.1)$ \\
\hline Persistent AF & $69(24.9)$ \\
\hline PVI only & $164(59.2)$ \\
\hline PVI + LA linear ablation & $76(27.4)$ \\
\hline Stepwise ablation & $37(13.4)$ \\
\hline VA & $216(14.4)$ \\
\hline Idiopathic VA & $184(12.3)$ \\
\hline
\end{tabular}

Table 1 continued

\begin{tabular}{ll}
\hline Characteristics & $\begin{array}{l}\text { Total number } \\
\text { of procedures } \\
(\boldsymbol{n}=\mathbf{1 5 0 0})\end{array}$ \\
\hline Structural VT & $32(2.1)$ \\
\hline
\end{tabular}

Data presented as median value (interquartile range) or numbers (percentage), unless otherwise stated

$A F$ atrial fibrillation, $A V$ atrioventricular, $A V N R T$ atrioventricular nodal reentrant tachycardia, $B M I$ body mass index, $E F$ ejection fraction, $L A$ left atrium, $P V I$ pulmonary vein isolation, $S V T$ supraventricular tachycardia, $V A$ ventricular arrhythmia, $V T$ ventricular tachycardia

of $569(37.9 \%)$ left-sided procedures were performed. In $163(28.6 \%)$ of the left-sided procedures we used transaortic access, while transseptal access was performed in 406 (71.4\%) procedures (the foramen ovale appertum was used in 41, while fluoroscopy-, TEE- or ICE-guided TSP was performed in 339, 24 and 2 procedures, respectively). Conventional RF or irrigated RF was used in $40.9 \%$ and $59.1 \%$ of the procedures, respectively.

There were no procedure-related deaths. Tamponade occurred during 12 (0.8\%) procedures, of which 10 were AF ablations (9 paroxysmal and 1 persistent AF ablation), 1 was idiopathic left-sided VA ablation, and 1 procedure was a typical AFL ablation. The prevalence of tamponade by the procedure type was $3.6 \%$ for AF ablation, $0.5 \%$ for VA ablation and $0.1 \%$ for SVT (non-AF) ablation. In 11 cases tamponade occurred in the index procedure and in 1 case during the redo procedure, and it never re-occurred in the same patient. On univariate analysis, the occurrence of tamponade was significantly more frequent in older patients, pre-procedure oral anticoagulation, left-sided procedures, 
Table 2 Predictors of cardiac tamponade

\begin{tabular}{|c|c|c|c|c|c|c|}
\hline \multirow[t]{2}{*}{ Predictors } & \multirow{2}{*}{$\begin{array}{l}\text { Procedures without } \\
\text { tamponade } \\
(n=1488)\end{array}$} & \multirow{2}{*}{$\begin{array}{l}\text { Procedures with } \\
\text { tamponade } \\
(n=12)\end{array}$} & \multicolumn{2}{|l|}{ Univariate analysis } & \multicolumn{2}{|c|}{ Multivariable analysis } \\
\hline & & & OR (95\% CI) & $P$ value & OR (95\% CI) & $P$ value \\
\hline Age (years) & $55.0(41.0-63.0)$ & $63.0(60.3-64.8)$ & $1.05(1.00-1.10)$ & 0.035 & - & - \\
\hline Males & $865(58.1)$ & $7(58.3)$ & $1.01(0.32-3.19)$ & 0.989 & - & - \\
\hline $\begin{array}{l}\text { Oral } \\
\text { anticoagulation } \\
\text { before } \\
\text { procedure }\end{array}$ & $447(30.0)$ & $11(91.7)$ & $25.62(3.30-119.02)$ & 0.002 & - & - \\
\hline $\begin{array}{l}\text { Left ventricular } \\
\text { EF (\%) }\end{array}$ & $60.0(56.0-66.0)$ & $63.5(61.0-66.5)$ & $1.00(0.99-1.02)$ & 0.546 & - & - \\
\hline $\begin{array}{l}\text { Left atrial } \\
\text { diameter }(\mathrm{mm})\end{array}$ & $38.0(34.0-43.0)$ & $40.0(36.5-44.5)$ & $1.02(0.95-1.10)$ & 0.543 & - & - \\
\hline Redo procedures & $243(16.3)$ & $1(8.3)$ & $0.47(0.07-3.03)$ & 0.430 & - & - \\
\hline $\begin{array}{l}\text { Transseptal } \\
\text { procedures }\end{array}$ & $396(26.6)$ & $10(83.3)$ & $13.79(3.01-63.20)$ & 0.001 & - & - \\
\hline $\begin{array}{l}\text { Left-sided } \\
\text { procedures }\end{array}$ & $558(37.5)$ & $11(91.7)$ & $18.33(2.36-142.38)$ & 0.005 & - & - \\
\hline AF ablation & $267(17.9)$ & $10(83.3)$ & $22.87(4.98-104.96)$ & $<0.001$ & $\begin{array}{l}13.86 \\
(2.78-69.04)\end{array}$ & 0.001 \\
\hline $\begin{array}{l}\text { SVT (non-AF) } \\
\text { ablation }\end{array}$ & $1006(67.6)$ & $1(8.3)$ & $0.04(0.01-0.34)$ & 0.003 & - & - \\
\hline VA ablation & $215(14.4)$ & $1(8.3)$ & $0.54(0.07-4.19)$ & 0.554 & - & - \\
\hline RF pulses $(n)$ & $17(8-33)$ & $44(23-55)$ & $1.01(1.00-1.03)$ & 0.074 & - & - \\
\hline RF time (min) & $15.5(8.2-34.4)$ & $52.1(19.3-64.7)$ & $1.01(1.00-1.03)$ & 0.106 & - & - \\
\hline $\begin{array}{l}\text { Energy } \\
\qquad(\times 1000 \mathrm{~W})\end{array}$ & $28.0(14.7-58.5)$ & $77.6(27.5-97.0)$ & $1.01(1.00-1.02)$ & 0.248 & - & - \\
\hline $\begin{array}{l}\text { Irrigated-tip } \\
\text { catheter }\end{array}$ & $875(58.8)$ & $12(100)$ & $7.71(1.00-59.85)$ & 0.051 & - & - \\
\hline $\begin{array}{l}\text { Fluoroscopy time } \\
\qquad(\min )\end{array}$ & $14.5(8.1-26.5)$ & $30.0(17.0-39.0)$ & $1.03(1.00-1.06)$ & 0.032 & - & - \\
\hline
\end{tabular}

Data shown as median (interquartile range) or numbers (percentage)

$A F$ atrial fibrillation, $E F$ ejection fraction, $O R$ odds ratio, $R F$ radiofrequency, $S V T$ supraventricular tachycardia; $V A$ ventricular arrhythmia

transseptal procedures, AF ablation, prolonged fluoroscopy, and irrigated-tip RF-CAs (Table 2). On multivariable analysis, only AF ablation (odds ratio: 13.86 , 95\% confidence interval: 2.78-69.04; $\quad P=0.001) \quad$ was significantly associated with tamponade. 


\section{Management and Outcome of Tamponade}

The perforation was attributed to catheter manipulation, excessive RF ablation and misguided TSP in five, five and two procedures, respectively. The perforation site was identified in the LA in seven, in the RA in three, in the left ventricle (LV) in one and in the CS in one procedure (Table 3 ).

In two cases, tamponade resulted in electromechanical dissociation and cardiorespiratory arrest (procedures 9 and 12; Table 3), while in the remaining ten patients tamponade was associated with significant hypotension (the mean systolic blood pressure at the moment of pericardial puncture was $59 \pm 18 \mathrm{mmHg}$ ). Pericardiocentesis was performed with the subxiphoid approach in 11 and with the transcardial approach in 1 patient (Table 4). Namely, in procedure 5, the LA roof was perforated with an ablative catheter during an attempted right superior PV angiography. The long steerable sheath (8.5 Fr) was left in the pericardial space for drainage purposes, while at the same time the re-isolation of lower PVs was completed and protamine was administered. The long sheath was replaced via the guidewire with an angiographic catheter of smaller diameter ( $5 \mathrm{Fr}$ ), and finally the guidewire itself was left in the pericardium for an approximate 30-min observation period before final removal after fluid accumulation cessation.

After pericardiocentesis, in ten patients $(83.3 \%)$, complete hemodynamic stability was achieved and the drainage catheter was removed after 6-48 h, while in two patients (16.7\%) urgent surgery was necessary because of continuous bleeding.

In procedure 9 (Tables 3,4 ), lateral PV isolation was complicated by the posterior LA wall perforation. After resuscitation and pericardiocentesis, the patient experienced painful abdomen distension with the sonographic image showing fluid collections in the abdominal cavity. To investigate suspected hollow organ perforation, emergency surgical abdomen exploration was performed, followed by thoracotomy with direct pericardial sac revision and fibrin glue application around the lateral PVs because of intraoperative suspicion of mediastinal bleeding. After 2 weeks of post-reanimation coma, the patient was treated for sepsis and finally recovered.

In procedure 12 (Tables 3,4 ), tamponade occurred during the LA linear endocardial ablation. Temporary hemodynamic recovery was achieved with pericardiocentesis; however, electromechanical dissociation developed quickly, and active drainage of the pericardium was continued during transportation to the cardiac surgery unit $(2830 \mathrm{ml}$ of blood was returned by auto-transfusion). Subsequently, INR spontaneously increased from 1.4 before ablation to 2.7 prior to surgery. The patient fully recovered after surgical mitral isthmus suture.

Overall, the mean amount of drained blood was $782 \pm 696 \mathrm{ml}$. Nine patients received blood transfusions (4 patients received only auto-transfusion, 2 were given only allogeneic transfusion, and 3 received both types of transfusion). The mean drop in hemoglobin after the procedure was $22 \pm 14 \mathrm{~g} / \mathrm{l}$.

Pericardiocentesis-related complications occurred in four $(33.3 \%)$ patients (two cases of clinically relevant pericarditis and two cases of septicemia). After pericardiocentesis, most patients were treated with anti-inflammatory drugs for chest pain (Table 4).

In six patients $(50.0 \%)$ with tamponade, the ablation was completed despite cardiac perforation. Redo ablation was subsequently 


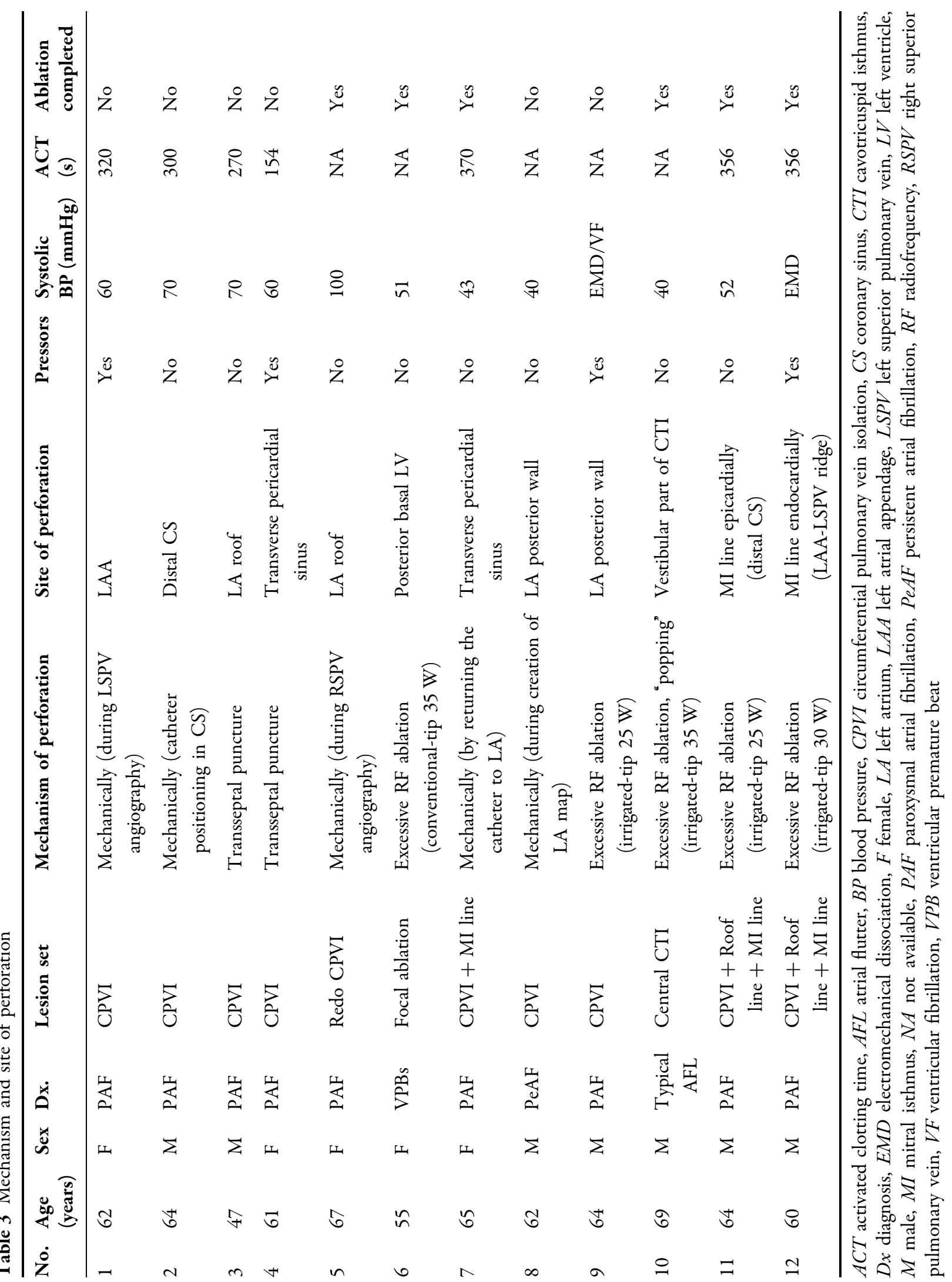




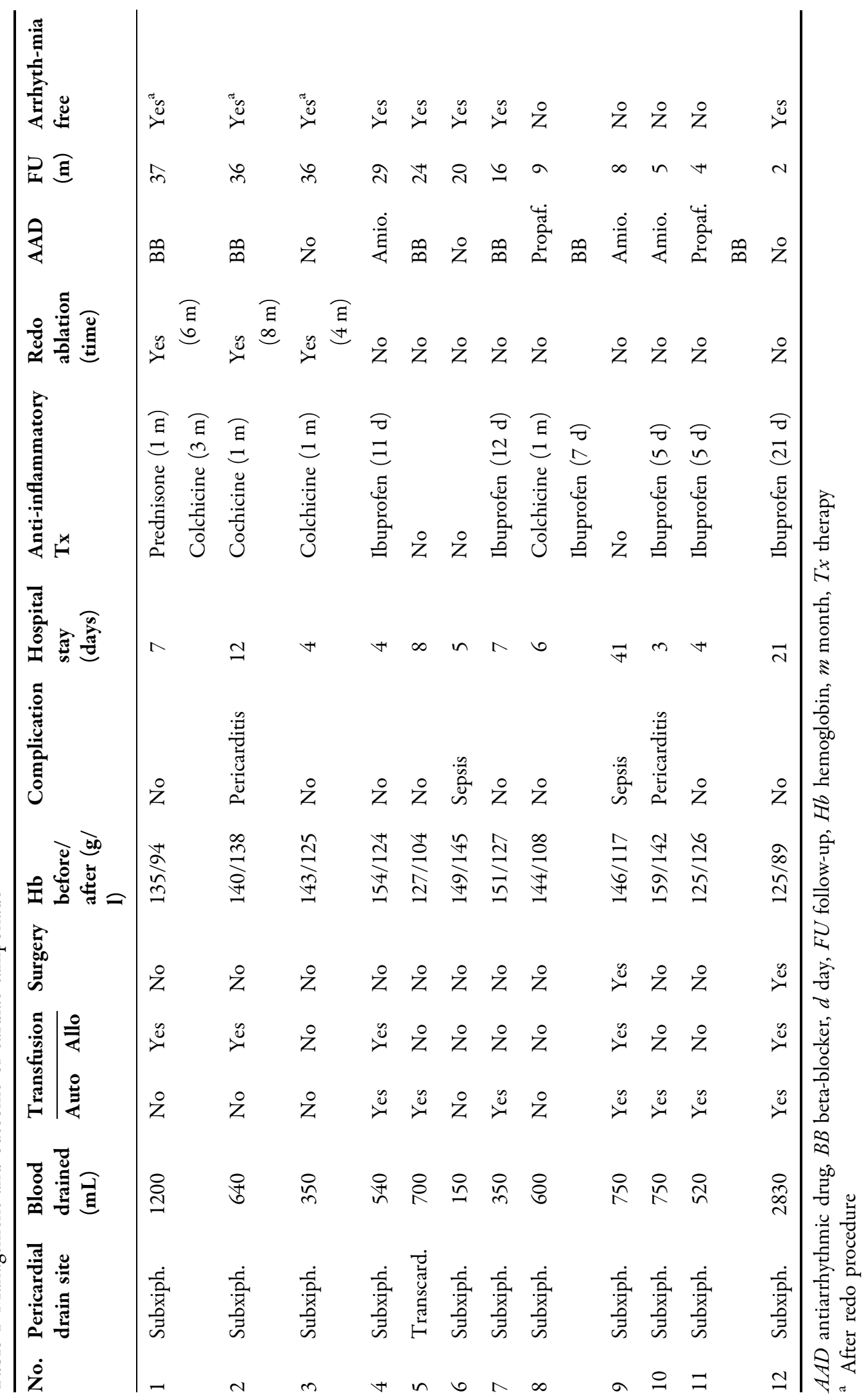


performed in three patients 4,6 and 8 months after the complication, respectively. During follow-up of $19 \pm 14$ months after ablation, eight $(66.7 \%)$ patients were arrhythmia free and nine patients (77\%) were on antiarrhythmic drugs (Table 4).

\section{DISCUSSION}

Our study of 1500 consecutive CA procedures performed in a medium-volume center considers a large data set outside the high-volume centers, showing relatively low overall incidence of CA-related tamponade $(0.8 \%)$, with no case fatality. Similar to the reports from high-volume centers, periprocedural tamponade in our center most commonly occurred with AF ablation (3.6\%) $[1,2,4,6,7]$.

\section{Risk Factors for Tamponade}

In our center, tamponade due to RF-CA was significantly more frequent in older patients, with pre-procedural oral anticoagulation, with a transseptal procedure, left-sided or AF ablation, with the use of irrigated-tip catheters and during the procedures with prolonged fluoroscopy. The only multivariable predictor of tamponade was AF ablation. Other reports additionally described VA ablation, female sex and the operator's (in)experience as the risk factor for RF-CA major complications [2, 6, 12]. Specific risks of tamponade during AF ablation are: redo procedure, linear LA ablation and use of $\mathrm{RF}$ power $>45 \mathrm{~W}[4,12,13]$.

The presumed mechanisms of perforation in our study were mechanical catheter manipulation, excessive tissue ablation and misdirected TSP. The use of a long steerable sheath improves catheter stability and ablation efficiency, but also enhances the catheter contact force to the tissue, increasing the risk of perforation [14]. The application of contemporary contact force measurement technology may reduce the perforation rate during RFCA [15].

TSP guided only by fluoroscopy is complicated by tamponade in $0.1-3.2 \%$ of cases $[5,10]$. ICE and TEE provide direct visualization of the septum and offer a safer TSP, especially in atypical anatomy, a resistant/ elastic septum and inexperienced operators $[16,17]$. Use of ICE during the AF ablation can provide many other potential benefits, such as the detection of LA thrombus or spontaneous echo-contrast formation, monitoring of catheter position and catheter-tissue contact, early identification of pericardial effusion during ablation, optimal RF energy titration and reduction of radiation exposure time [17-19]. However, routine application of TEE requires general anesthesia in many patients and may contribute to esophageal injury during AF ablation [20], while use of ICE increases the cost of the procedure [21].

More intensive anticoagulation and higher intracavital pressure increase the risk of tamponade during left-sided procedures [5]. Therefore, it is not surprising that in our study the majority of tamponade cases occurred as a complication of a left-sided AF ablation.

Tamponade is the most frequent cause of fatal AF ablation outcome and is responsible for a quarter of all procedure-related deaths [3]. It has been shown that AF ablation carries a significantly higher risk of perforation compared with other transseptal procedures, such as left-sided AP ablation or balloon mitral valvuloplasty [22]. Dual transseptal access, prolonged LA catheter manipulation time, a thin LA wall, necessity of transmural and extensive ablation as well as irrigated-tip catheter use may explain a higher tamponade 
rate in $\mathrm{AF}$ ablation in comparison to other types of procedures in the present study. In comparison to RF conventional-tip catheters, externally irrigated-tip catheters create a deeper and wider lesion and increase the risk of tissue overheating and "steam-pop" [4, 13].

\section{Perforation Predilection Sites}

Thinned LA wall segments (the appendage, vestibular part of the mitral annulus, posterior roof), the RV apex and distal CS represent perforation predilection sites during EP procedures [5, 7, 23].

In a study of 15 patients with $\mathrm{AF}$ ablation-related tamponade, the LA was perforated in nine, the RV in five and the RA in one patient [7]. Likewise, in our study, the LA perforation most frequently occurred during $\mathrm{AF}$ ablation (in 7 out of 10 patients with $\mathrm{AF}$ and tamponade). As opposed to other studies, there were no RV perforations in the present study, most probably because of the simplified catheter setup during AF ablation as well as the absence of a RV stimulating catheter. In our study, one procedure of typical AFL ablation led to RA perforation. Although the cavo-tricuspid isthmus ablation is one of the safest procedures, this complication has also been recognized by other authors [4].

Aggressive irrigated RF ablation, systemic anticoagulation and higher intracavital pressure are responsible for a significant risk of tamponade accompanying VA ablation $[2,8]$. Additionally, the differences in the wall thickness are responsible for more frequent perforation of the $\mathrm{RV}$ as compared to the $\mathrm{LV}$ [5]. In a series of 892 patients subjected to RFCA of VA, tamponade complicated 11 procedures (1.23\%) [8]. The laceration site was localized in the RV in seven, in the LV in only one and in three patients it remained undetermined. In the present study, there was only one tamponade due to VA ablation, this being an idiopathic VA in the LV basal inferior wall segment. It is possible that transaortic access to the $\mathrm{LV}$ inferior wall provides a greater contact force and perpendicular catheter tip orientation, predisposing the myocardium to perforation during RF [13]. A low tamponade rate in the subpopulation of patients with VA (0.46\%) could be explained by our practice of careful RF energy titration led by the impedance drop of $\leq 10 \Omega$. Namely, in VA ablation, the decrease of impedance $\geq 18 \Omega$ was associated with a significantly more frequent occurrence of "steam-pop" and perforation [8].

\section{Tamponade Management and Outcome}

For pericardiocentesis, it is optimal to choose the shortest and safest route of the needle from the skin to the pericardium, the direction of the largest pericardial fluid accumulation and a technique the operator is most familiar with [5]. In one study, the echocardiogram-guided apical approach was predominant (66.7\%) [7], while in another study, the fluoroscopy-guided subxiphoid approach was mostly used (83.3\%) [8], which is similar to our experience. In our study, subxiphoid access to the pericardium was achieved in the left lateral fluoroscopic projection, which clearly defines the path of the needle during puncture and best separates mediastinal, pericardial and myocardial structures [11]. However, the subxiphoid access can be complicated by perforation of the RV, stomach, colon or liver [5, 7, 8]. Indeed, one of our patients underwent an urgent surgical abdomen exploration after pericardiocentesis because of suspected hollow organ perforation.

Life-saving pericardiocentesis can be performed via the transcardial approach, by 
the introduction of a long sheath over the catheter or by a needle through the myocardium into the pericardial space to achieve emergency drainage [23]. After fluid evacuation, it is serially replaced with angiographic catheters of gradually smaller lumens until cessation of bleeding or surgical management [23]. A similar experience in the present study was noted.

The probability of spontaneous bleeding cessation after pericardiocentesis depends on the site, size and geometry of the perforation, intracavital pressure and anticoagulation level [5]. Continuous bleeding after pericardiocentesis requires urgent surgery. Previous studies have shown that only $13 \%$ of patients after atrial perforation and as much as $55 \%$ of patients after ventricular perforation underwent cardiac surgery [7, 8]. Similarly, in our study two (16.7\%) patients were surgically treated after pericardiocentesis. In both cases the LA perforation caused tamponade. Since the number of AF ablation procedures is increasing, the necessity of intra-hospital cardiac surgery support is becoming an important issue. We believe that this should be emphasized because $>50 \%$ of the centers currently perform AF ablations without in-house surgical back-up [24].

In the present study auto-transfusion was carried out in seven patients. Direct auto-transfusion is simple and requires no additional equipment; it may abolish the need for allogeneic blood transfusion and can "buy" time until surgery [23]. However, direct auto-transfusion may cause systemic inflammation, and therefore the processing of the drained blood via the cell salvage system is recommended prior to its return [25]. Auto-transfusion of a larger volume of blood, i.e., more than $1500 \mathrm{ml}$, may lead to consumptive coagulopathy [23, 25], which occurred in one patient in our study. In such circumstances, a timely replacement of coagulation factors and thrombocytes, an infusion of freshly frozen plasma or a whole-blood transfusion is necessary $[23,25]$.

In half of our patients with tamponade, the planned ablation set of lesions was completed despite perforation. In case of a smaller perforation, slow pericardial blood accumulation left sufficient time for the ablation to be completed before the development of tamponade; in another patient, a larger laceration led to massive hemorrhage and a sudden collapse soon after final "successful" RF application. Our study has also confirmed that ablation can be safely repeated after 4-8 months and, despite serious complications, in two-thirds of the patients it was possible to finally achieve arrhythmia elimination [7].

\section{Limitations}

This study included a 5-year period, and the learning curve of individual operators might have significantly influenced the rate of complications. In addition, the mechanism and site of perforation in un-operated patients were determined indirectly. In our study, ICE and TEE were used in only $7.7 \%$ of transseptal procedures. It is possible that the routine use of ICE or TEE during the AF ablation procedure could significantly reduce the rates of cardiac tamponade and other mechanical complications. In the current study, AF ablation was performed with an interrupted warfarin strategy. Although an uninterrupted warfarin strategy reduces rates of thromboembolic stroke and minor bleeding in this setting, it deviates from the local surgical practice and has not been implemented in $40 \%$ of low-to-medium volume EP centers in Europe [26]. 


\section{CONCLUSIONS}

In our study of a medium-volume center, the incidence of RF-CA-related cardiac tamponade was less than $1 \%$, and its occurrence was significantly associated with AF ablation. The outcome of tamponade was mostly favorable after pericardiocentesis, but readily available cardiothoracic surgery backup was necessary in one of five to six procedure-related tamponade cases.

\section{ACKNOWLEDGMENTS}

No funding or sponsorship was received for this study or publication of this article. All named authors meet the International Committee of Medical Journal Editors (ICMJE) criteria for authorship for this manuscript, take responsibility for the integrity of the work as a whole and have given final approval to the version to be published.

Disclosures. Nebojša Mujović, Milan Marinković, Nebojša Marković, Aleksandar Kocijančić, Vladan Kovačević, Dragan Simić, Arsen Ristić, Goran Stanković, Biljana Miličić, Svetozar Putnik, Bosiljka Vujisić-Tešić and Tatjana S. Potpara declare that they have no conflict of interest.

Compliance with Ethics Guidelines. The study was conducted in accordance with the Declaration of Helsinki of 1964 as revised in 2013. The study protocol was approved by the Institutional Review Board of our hospital. This article is based on previously conducted studies and does not involve any new studies of human or animal subjects performed by any of the authors.
Open Access. This article is distributed under the terms of the Creative Commons AttributionNonCommercial 4.0 International License (http://creativecommons.org/licenses/by-nc/4. $0 /$ ), which permits any noncommercial use, distribution, and reproduction in any medium, provided you give appropriate credit to the original author(s) and the source, provide a link to the Creative Commons license, and indicate if changes were made.

\section{REFERENCES}

1. Cappato R, Calkins H, Chen SA, et al. Updated worldwide survey on the methods, efficacy, and safety of catheter ablation for human atrial fibrillation. Circ Arrhythm Electrophysiol. 2010;3:32-8.

2. Bohnen M, Stevenson WG, Tedrow UB, et al. Incidence and predictors of major complications from contemporary catheter ablation to treat cardiac arrhythmias. Heart Rhythm. 2011;8:1661-6.

3. Cappato R, Calkins H, Chen SA, et al. Prevalence and causes of fatal outcome in catheter ablation of atrial fibrillation. J Am Coll Cardiol. 2009;53:1798-803.

4. Jaïs P, Hocini M, Hsu LF, et al. Technique and results of linear ablation at the mitral isthmus. Circulation. 2004;110:2996-3002.

5. Holmes JRDR, Nishimura R, Fountain R, Turi ZG. Iatrogenic pericardial effusion and tamponade in the percutaneous intracardiac intervention era. J Am Coll Cardiol Intv. 2009;2:705-17.

6. Michowitz Y, Rahkovich M, Oral H, et al. Effects of sex on the incidence of cardiac tamponade after catheter ablation of atrial fibrillation. Results from a worldwide survey in 34943 atrial fibrillation ablation procedures. Circ Arrhythm Electrophysiol. 2014;7:274-80.

7. Bunch TJ, Asirvatham SJ, Friedman PA, et al. Outcomes after cardiac perforation during radiofrequency ablation of the atrium. J Cardiovasc Electrophysiol. 2005;16:1172-9. 
8. Tokuda $\mathrm{M}$, Kojodjojo $\mathrm{P}$, Laurence $\mathrm{M}$, et al. Outcomes of cardiac perforation complicating catheter ablation of ventricular arrhythmias. Circ Arrhythm Electrophysiol. 2011;4:660-6.

9. Yao Y, Ding L, Chen W, et al. The training and learning process of transseptal puncture using a modified technique. Europace. 2013;15:1784-90.

10. De Ponti R, Cappato R, Curnis A, et al. Trans-septal catheterization in the electrophysiology laboratory: data from a multicenter survey spanning 12 years. J Am Coll Cardiol. 2006;47:1037-42.

11. Weerasooriya R, Jaïs P, Sacher F, et al. Utility of the lateral fluoroscopic view for subxiphoid pericardial access. Circ Arrhythm Electrophysiol. 2009;2:e15-7.

12. Baman TS, Jongnarangsin $\mathrm{K}$, Chugh A, et al. Prevalence and predictors of complications of radiofrequency catheter ablation for atrial fibrillation. J Cardiovasc Electrophysiol. 2011;22:626-31.

13. Yuyun MF, Stafford PJ, Sandilands AJ, Samani NJ, $\mathrm{Ng}$ GA. The impact of power output during percutaneous catheter radiofrequency ablation for atrial fibrillation on efficacy and safety outcomes: a systematic review. J Cardiovasc Electrophysiol. 2013;24:1216-23.

14. Matsuo S, Yamane T, Date T, et al. Completion of mitral isthmus ablation using a steerable sheath: prospective randomized comparison with a nonsteerable sheath. J Cardiovasc Electrophysiol. 2011;22:1331-8.

15. Nazeri A, Ganapathy A, Massumi A, et al. Contact-force recovery can predict cardiac perforation during radiofrequency ablation. Pacing Clin Electrophysiol. 2014;37:1129-32.

16. Bayrak F, Chierchia GB, Namdar M, et al. Added value of transoesophageal echocardiography during transseptal puncture performed by inexperienced operators. Europace. 2012;14:661-5.

17. Saliba W, Thomas J. Intracardiac echocardiography during catheter ablation of atrial fibrillation. Europace. 2008;10(Suppl 3):iii42-7.

18. Ren JF, Marchlinski FE, Callans DJ, et al. Increased intensity of anticoagulation may reduce risk of thrombus during atrial fibrillation ablation procedures in patients with spontaneous echo contrast. J Cardiovasc Electrophysiol. 2005;16:474-7.

19. Ferguson JD, Helms A, Mangrum JM, et al. Catheter ablation of atrial fibrillation without fluoroscopy using intracardiac echocardiography and electroanatomic mapping. Circ Arrhythm Electrophysiol. 2009;2:611-9.

20. Kumar S, Brown G, Sutherland F, et al. The transesophageal echo probe may contribute to esophageal injury after catheter ablation for paroxysmal atrial fibrillation under general anesthesia: a preliminary observation. J Cardiovasc Electrophysiol. 2015;26:119-26.

21. Winkle RA, Mead RH, Engel G, Kong MH, Patrawala RA. Physician-controlled costs: the choice of equipment used for atrial fibrillation ablation. J Interv Card Electrophysiol. 2013;36:157-65.

22. Katritsis GD, Siontis GC, Giazitzoglou E, Fragakis N, Katritsis DG. Complications of transseptal catheterization for different cardiac procedures. Int J Cardiol. 2013;168:5352-4.

23. O’Neill MD, Jaïs P, Derval N, Hocini M, Haïssaguerre M. Two techniques to avoid surgery for cardiac tamponade occurring during catheter ablation of atrial fibrillation. J Cardiovasc Electrophysiol. 2008;19:323-5.

24. Neuberger HR, Tilz RR, Bonnemeier $\mathrm{H}$, et al. A survey of German centres performing invasive electrophysiology: structure, procedures, and training positions. Europace. 2013;15:1741-6.

25. Venkatachalam KL, Fanning LJ, Willis EA, et al. Use of an autologous blood recovery system during emergency pericardiocentesis in the electrophysiology laboratory. J Cardiovasc Electrophysiol. 2009;20:280-3.

26. Chen J, Todd DM, Hocini M, Larsen TB, Bongiorni MG, Blomström-Lundqvist C. Current periprocedural management of ablation for atrial fibrillation in Europe: results of the European Heart Rhythm Association survey. Europace. 2014;16:378-81. 